\title{
Instrumento para classificação de recém-nascidos de acordo com o grau de dependência de cuidados de enfermagem*
}

\author{
Instrument for classification of neonates in according of \\ dependence degree of nursing's care
}

\author{
Instrumento para clasificación de bebés de acuerdo con el grado \\ de dependencia y de los cuidados de enfermaría
}

Luciana Bochembuzio', Raquel Rapone Gaidzinski²

\begin{abstract}
RESUMO
Objetivo:Trata-se da reconstrução de um instrumento para classificação de recém-nascidos proposto por Toledo e Fugulin em 2000. Métodos:Foram consideradas 16 áreas de cuidado de enfermagem e o neonato foi classificado em três níveis de complexidade assistencial. Para a validação do conteúdo, utilizou-se a técnica Delphi. Participaram como juízas cinco enfermeiras da área obstétrica, neonatal e pediátrica que atuam junto a instituições assistenciais ou vinculadas a Hospital-Escola. Resultados:Os resultados obtidos mostraram concordância das juízas quanto a: manutenção das 16 áreas de cuidado, a pertinência e clareza das áreas e a existência de nível crescente de complexidade assistencial.
\end{abstract}

Descritores: Recém-nascido; Enfermagem neonatal; Enfermagem pediátrica

\begin{abstract}
Objective:To deal about reconstruction of the instrument for classification of neonates proposed for Toledo and Fugulin in 2000. Methods:It was considered 16 caring areas and the neonate classified in three levels of the nursing care complexity. For the content validation was utilized the Delphi technique. A team of fivs experts nursing professionals of neonate, obstetric and pediatric area whom attends patients or teaches in the Medical School. Results:The obtained results have showed the experts agreement concerned to: the maintenance of the 16 caring area, property and intelligibility of the area contents and presence of an increasing level in the nursing care complexity.
\end{abstract}

Keywords: Infant, newborn; Neonatal nursing; Pediatric nursing

\section{RESUMEN}

Objetivo: Este artículo trata sobre la reconstrucción de un instrumento para clasificación de neonatos propuesto por Toledo y Fugulin en año 2000. Métodos: Fueron consideradas 16 áreas de cuidado de enfermería, clasificándose al neonato en tres niveles de complejidad asistencial. Para a validación del contenido, se utilizó la técnica Delphi. Participaron como jueces cinco enfermeras del área obstétrica, neonatal y pediátrica que trabajan en instituciones asistenciales o vinculadas a un Hospital Docente. Resultados:Los resultados obtenidos mostraron concordancia de las jueces en cuanto a: manutención de las 16 áreas de cuidado, la pertinencia y claridad de las áreas y la existencia de un nivel creciente de complejidad asistencial.

Descriptores: Recién nacido; Enfermería neonatal; Enfermería pediátrica

\footnotetext{
* Trabalho extraído da Dissertação de Mestrado defendidana Escola de Enfermagem da Universidade de São Paulo - USP - São Paulo (SP), Brasil, em 2002.

1 Mestre em Enfermagem pela Universidade de São Paulo - USP - São Paulo(SP), Brasil

2 Professora Associada do Departamento de Orientação Profissional da Escola de Enfermagem da Universidade de São Paulo - USP - São Paulo(SP), Brasil
} 


\section{INTRODUÇÃO}

A preocupação com a assistência ao recém-nascido (RN) na área da saúde surgiu como um prolongamento da ação da prática obstétrica. Recursos materiais, humanos e terapêuticos se faziam necessários para o controle da termorregulação, alimentação e prevenção de infecção, além de planta física e dispositivos próprios para o atendimento, como incubadoras, procedimentos e normas rigorosas de isolamento e treinamento especial do pessoal ${ }^{(1)}$. Os berçários tinham a finalidade social de diminuir a mortalidade infantil dos países industrializados, visando a força de trabalho e evitando mortes prematuras, tendo em vista a diminuição da natalidade e o risco do despovoamento.

Não há um estudo sistemático da história da organização dos berçários nas instituições hospitalares no cenário nacional. No Brasil, apesar dos berçários distribuírem os RNs sob áreas de cuidados progressivos, não existe, na maioria das instituições, uma definição formal que contemple os aspectos globais da assistência em cada área e que seja passível de ser utilizada no contexto multiprofissional, isto é, que possibilite a construção de uma base comum de interpretação para a avaliação e classificação dos pacientes e favoreça a complementariedade das ações assistenciais ${ }^{(2)}$.

Em 2000, Toledo e Fugulin elaboraram um instrumento com a finalidade de contribuir para a consolidação de uma base comum de avaliação e classificação dos RNs de acordo com as necessidades de cuidado de enfermagem ${ }^{(2)}$. Nesse estudo proponho-me a reestruturar esse instrumento com a perspectiva de torná-lo mais adequado às unidades neonatais no contexto nacional. Esse instrumento reestruturado tem a função de orientar a classificação de recém-nascidos por tipo de cuidado com base nas necessidades individualizadas de assistência de enfermagem, e contribuir para a qualidade do atendimento, assegurando que os RNs não sejam lesados em seus direitos diante da vida ${ }^{(3)}$.

Assim, este estudo se propõe a:

- reconstruir o instrumento para classificação de pacientes em unidade neonatal, proposto por Toledo e Fugulin $^{(2)}$ em 2000, de acordo com as necessidades assistenciais de enfermagem.

- validar o conteúdo do novo instrumento de classificação de pacientes com as juízas especialistas, utilizando a técnica Delphi ${ }^{(4)}$.

\section{O SISTEMA DE CLASSIFICAÇÃO PARA RECÉM-NASCIDOS}

A implantação e o desenvolvimento do sistema de classificação de pacientes (SCP) para a área neonatal pode favorecer o estabelecimento do vínculo afetivo entre o trinômio mãe-pai-filho, incentivar o aleitamento natural flexível, de acordo com as necessidades dos RNs, possibilitar a redução da incidência de infecção hospitalar, permitir que a mãe receba orientação adequada e desenvolva capacidades para cuidar de seu filho, considerando suas limitações e as do recém-nascido.

A Organização Pan Americana de Saúde/Organização Mundial de Saúde (OPAS/OMS) sugere a adoção de um sistema de classificação de pacientes quando se preocupa em implementar e garantir a qualidade nos hospitais latino-americanos, propondo a utilização de programas de Acreditação. Em seu manual, menciona a Categorização como um método criterioso a ser utilizado para a classificação de serviços ${ }^{(5)}$.

A Resolução Conselho Federal de Enfermagem (COFEN), número $189 / 96^{(6)}$ foi instituída considerandose a inexistência de uma norma que padronizasse a relação profissionais/leitos para a assistência de enfermagem ao paciente, estabelecendo parâmetros mínimos para o dimensionamento do quadro de profissionais de enfermagem nas instituições de saúde. Este documento determina que é de competência do enfermeiro quanti e qualificar o quadro de profissionais necessário para a prestação de assistência ${ }^{(7)}$. E, esta estimativa de pessoal deve ser embasada no SCP que será determinado pelos diferentes tipos de cuidados: mínimo ou autocuidado; intermediário; semi-intensivo e intensivo, de acordo com o perfil de cuidados ${ }^{(8)}$.

Na grande maioria dos berçários das instituições hospitalares brasileiras a avaliação, a classificação dos pacientes e a definição da área a ser encaminhado o RN é realizada pelo pediatra ou neonatologista no Centro Obstétrico com base no peso do nascimento, na Idade Gestacional, no valor da avaliação do Capurro Somático e em normas e condutas que definem patologias ou quadros clínicos de cada nascituro. Do mesmo modo, o dimensionamento do pessoal de enfermagem que presta cuidados aos neonatos não é baseado num sistema de classificação de pacientes, mas em critérios administrativos peculiares a cada instituição.

Dentro do trabalho coletivo de assistência em unidade neonatal, a equipe de enfermagem tem se responsabilizado pelo cuidado direto e contínuo do recémnascido e pela orientação dos pais sobre os cuidados com a criança, assim como tem assumido a gerência da unidade de internação e controle do ambiente. A assistência está organizada de diferentes formas: em alguns serviços cada médico determina os procedimentos a serem executados do nascimento à alta hospitalar do neonato e em outros há uma padronização de condutas e normas escritas. Esta normatização de procedimentos e ações, em geral, é determinada por um fator de risco biológico materno ou neonatal próprio da rotina do serviço hospitalar ${ }^{(1)}$. 
A Academia Americana de Pediatria preconiza 6 áreas funcionais para o cuidado hospitalar do neonato ${ }^{(9)}$ área para Ressuscitação, área de Admissão/Observação, área de Cuidado do RN Normal, área de Cuidado Contínuo, área de Cuidado Intermediário e área de Cuidado Intensivo.

Nos últimos anos, além da melhoria dos cuidados ao binômio mãe-filho, através do parto hospitalar, berçário e alojamento conjunto, estabeleceu-se um importante conceito, que é o do atendimento perinatal em níveis hospitalares crescentes de complexidade, denominados nível I ou de Cuidados Mínimos, nível II ou de Cuidados Intermediários, nível III ou de Cuidados Intensivos, instalando-se o nível III em regiões estrategicamente situadas, para onde possam fluir os casos mais graves, isto é, regionalizando-se a assistência perinatal ${ }^{(10)}$.

O manual do Ministério da Saúde ${ }^{(11)}$, através da Secretaria de Assistência à Saúde, preconiza que a organização da assistência neonatal deve basear-se num sistema que garanta cuidados contínuos e de complexidade assistencial em relação proporcional ao nível de risco do neonato. Dessa maneira, para cada momento, desde o nascimento até a alta neonatal, devem ser definidas e planejadas as atividades assistenciais, considerando: o nível de cuidados do paciente, a área assistencial, os recursos humanos necessários, seu treinamento básico, as normas assistenciais e os procedimentos a serem utilizados.

Baseados em experiências práticas, vários autores da área neonatal ${ }^{(10-13)}$ sugerem padrões de assistência que estejam de acordo com a filosofia do serviço, estrutura organizacional e avaliação criteriosa dos recursos materiais e humanos disponíveis e se propõem a aplicar a sistematização da assistência para o desenvolvimento das atividades.

Não existe um instrumento formal que avalie, no contexto multiprofissional, as condições de nascimento do RN, que vislumbre um sistema de identificação e contribuição para o cuidado individualizado de enfermagem para grupos de neonatos com necessidades específicas, assegurando maior efetividade e produtividade do pessoal de enfermagem.

A implantação de um SCP em uma Unidade Neonatal seria a oportunidade de aperfeiçoar os procedimentos, as ações e as intervenções, redirecionando os processos de gestão, promovendo uma forma diferenciada de gerenciar recursos humanos, alocar recursos financeiros e definir necessidades de novas tecnologias, centradas no RN e na família, consolidando o conhecimento e a tecnologia de domínio profissional.

A consolidação da tecnologia com a assistência à saúde redireciona o cuidado para as modernas práticas de gestão participativa, centradas nos múltiplos padrões biológicos, psicológicos, sociais, cuidativos, culturais e educacionais, enquanto indicação de valor para a intervenção profissional, centrada na qualidade ${ }^{(14) .}$

\section{MÉTODOS}

Para este estudo, considerando a natureza do tema, optou-se por uma metodologia quantitativa descritiva.

Os sujeitos desta pesquisa foram cinco enfermeiras especialistas em Enfermagem Obstétrica, Neonatal e Pediátrica que julgaram o instrumento, considerando o conhecimento, a experiência e a vivência na área neonatal de cada uma delas.

A validação do instrumento de classificação de pacientes compreendeu três etapas: a reestruturação do instrumento de classificação de pacientes proposto por Toledo e Fugulin ${ }^{(2)}$ em 2000; a validação do conteúdo do instrumento de classificação de pacientes pela técnica Delphi ${ }^{(15)}$ e o estabelecimento do método para a padronização do escore. A técnica Delphi ${ }^{(15)}$ foi o processo utilizado para fundamentar o trabalho de campo dentro da perspectiva quantitativa descritiva.

\section{O Instrumento para classificação de recém-nascidos}

Para compor o instrumento os autores ${ }^{(2)}$ identificaram o perfil dos RNs, de acordo com as necessidades assistenciais de enfermagem e definiram as categorias de cuidado. Dessa forma, foram consideradas as seguintes áreas de cuidados: termorregulação, peso, atividade espontânea, reação à estímulos, cor da pele, tonicidade muscular, nutrição e hidratação, oxigenação, integridade cutâneo-mucosa, controle de sinais vitais Temperatura (T), Frequiência Respiratória (FR), Freqüência Cardíaca (FC), Pressão Arterial (PA), Saturação de $\mathrm{O}_{2}$, controle de drenos, sondas e cateteres, terapêutica medicamentosa.

Após a identificação do perfil dos pacientes, as autoras definiram os setores de cuidado por complexidade assistencial, observando as áreas de cuidado já determinadas para cada setor: baixo risco, médio risco, alto risco ou semi - intensivo, terapia intensiva.

No sentido de complementar o instrumento a pesquisadora acrescentou três áreas de cuidado: eliminações, cuidado corporal e educação à saúde e desconsiderou a área de cuidado denominada baixo risco, além de substituir a nomenclatura para cuidados mínimos, intermediários e intensivos.

\section{Validação do instrumento}

Optou-se pela aplicação da técnica Delphi ${ }^{(15)}$ para a validação do conteúdo do instrumento de classificação para que fosse possível avaliar a extensão da dependência do bebê com relação à enfermagem. 
A técnica Delphi é uma estrutura teórica utilizada para embasar e garantir a validade do conteúdo do estudo. Ela valoriza a participação de indivíduos conhecedores profundos de um determinado assunto e facilita a obtenção de resultados produtivos ${ }^{(15)}$.

Nesta pesquisa, a técnica Delphi foi aplicada em duas fases, seguindo as etapas:

Elaboração dos critérios para seleção dos juizes - foram convidadas cinco enfermeiras que correspondiam aos seguintes critérios: ter pelo menos cinco anos de experiência profissional em unidade neonatal; atuar junto a instituições de assistência ou vinculados a hospitalescola na cidade de São Paulo, aceitar participar do estudo na condição de juiz especialista.

Elaboração do questionário - o questionário foi composto de dados referentes à identificação, área de atuação e qualificação profissional dos juizes e por duas partes relativas à reestruturação do instrumento de classificação de recém-nascidos. Na parte I foram apresentadas 15 áreas de cuidado e os setores de cuidado por complexidade assistencial que compõem o instrumento, cabendo às juízas, através de questões fechadas em duas alternativas, SIM e NÃO, julgarem a pertinência de cada indicador no instrumento. Após estas alternativas reservou-se um espaço para que as enfermeiras registrassem os comentários que julgavam necessários. Na parte II do questionário, as juízas puderam apreciar a classificação dos indicadores no que diz respeito ao conteúdo, seguimento de um nível de complexidade assistencial crescente e clareza nos enunciados, assinalando SIM ou NÃO para cada um dos tópicos. Nesta fase, por sugestão de uma das juízas, foi acrescentada uma área de cuidado perfazendo um total de 16 áreas. Destinou-se, também, um espaço para que elas acrescentassem algum comentário pertinente. O questionário foi acompanhado de uma carta que esclarecia os objetivos da pesquisa, bem como as atividades solicitadas às juízas, pela pesquisadora. Estes questionários foram entregues, pela pesquisadora, estabelecendo um prazo de sete dias para o retorno.

Estabelecimento do índice de concordância para as respostas obtidas - foram considerados validados os indicadores que obtiveram índice de concordância nas respostas das juizas maior ou igual a $70 \%$.

Tabulação dos dados obtidos e análise da opinião convergente das juizas - no primeiro questionário os dados foram tabulados e tratados percentualmente para verificar se o índice de concordância encontrado foi maior ou igual a $70 \%$, tanto na parte relacionada ao número de áreas, como na parte relacionada ao conteúdo, nível de complexidade assistencial crescente e clareza.
Para se afirmar que um instrumento para classificação de pacientes é válido, é necessário que ele passe por um processo que permita concluir se os indicadores de cuidado representam, de fato, o que é encontrado na prática clínica ${ }^{(16)}$.

No que diz respeito aos aspectos éticos envolvidos na realização dessa pesquisa, cumpre esclarecer que a coleta de informações somente foi iniciada após parecer favorável do Comitê de Ética em Pesquisa da Escola de Enfermagem da Universidade de São Paulo, através da deliberação CEP/EEUSP processo $n^{\circ} 233 / 2002$.

\section{Método para a padronização do escore}

Toledo e Fugulin não estabeleceram escores como forma de atribuir valores aos setores de cuidado por complexidade assistencial em seu instrumento ${ }^{(2)}$

Para o instrumento reconstruído cada uma das 16 áreas de cuidado receberam valores de $1 \mathrm{a} 3$, a pontuação mínima alcançada é de 16 e a máxima de 48 pontos com amplitude total igual a 32 pontos. Os pontos foram distribuídos em 3 categorias correspondentes aos tipos de cuidados: mínimos, intermediários e intensivos. Definindo-se como:

- Cuidados mínimos - recém-nascidos estáveis sob ponto de vista clínico e de enfermagem, que permanecem em observação para a detecção de possíveis intercorrências ou de patologias que podem surgir nas primeiras horas ou dias de vida.

- Cuidados intermediários - recém-nascidos estáveis sob ponto de vista clínico e de enfermagem, sem risco de vida, mas que apresentam algumas intercorrências ou patologias que necessitam de cuidados médicos e de enfermagem específicos e permanentes para minimizar ou corrigir distúrbios hemodinâmicos decorrentes de suas patologias.

- Cuidados intensivos - recém-nascidos instáveis sob ponto de vista clínico e de enfermagem, com distúrbios hemodinâmicos importantes, com risco iminente de vida, que necessitam de cuidados médicos e de enfermagem específicos e permanentes para sua estabilização.

Desta forma, obteve-se um escore padronizado à cada categoria de cuidado, conforme esquema abaixo:

\begin{tabular}{c|c} 
TIPO DE CUIDADO & PONTUAÇÃO \\
\hline Mínimo & 16 a 26 pontos \\
Intermediário & 27 a 37 pontos \\
Intensivo & 38 a 48 pontos
\end{tabular}


Os escores clínicos têm como objetivo avaliar a gravidade da doença, a instabilidade clínica do RN, isto é, prognosticar. Esta avaliação procura enfatizar mais o curso natural da doença do que os mecanismos fisiopatológicos específicos da doença e sua recuperação ${ }^{(17)}$.

\section{APRESENTAÇÃO E DISCUSSÃO DOS RESULTADOS}

O grupo de juízas se manteve durante toda a pesquisa composto por cinco enfermeiras, todas do sexo feminino, das quais duas assistem diretamente recém-nascidos internados em unidade neonatal, duas atuam no ensino de enfermagem em cursos de graduação e/ou pós-graduação e uma trabalha na gerência de uma unidade neonatal.

A idade das enfermeiras variou de 31 a 48 anos. Todas têm entre 10 e 20 anos de experiência e sempre atuaram na área de Enfermagem Neonatal.

No que concerne à qualificação profissional, duas têm habilitação em Enfermagem Obstétrica e Neonatal; duas fizeram especialização em Enfermagem Pediátrica e Neonatal, uma em Enfermagem Obstétrica e uma delas fez especialização em Enfermagem Pediátrica e Neonatal e em Administração Hospitalar. Uma das enfermeiras está cursando especialização em Terapias Florais; quanto à pós-graduação, uma tem o doutorado concluído e a outra está em fase de conclusão.

Após a devolução dos questionários da parte I e II, as respostas foram analisadas quantitativamente e as considerações das juízas devidamente anotadas. O novo instrumento - instrumento ${ }^{(2)}$ elaborado por Toledo e Fugulin em 2000 e adaptado $^{(3)}$ em 2002 por Bochembuzio- foi descrito a partir do levantamento da literatura, da vivência da pesquisadora e da apreciação das juízas, sendo fundamentado nas necessidades humanas básicas ${ }^{(18)}$.

Cada etapa do estudo Delphi ${ }^{(15)}$ compreendeu a análise parcial dos resultados, a busca à convergência das respostas e a coerência entre as juízas para determinar o melhor instrumento para classificação de recém-nascidos.

Nesta pesquisa houve consenso entre as juízas com apenas duas rodadas de correspondências. Cada rodada de correspondência atendeu a previsão de até três semanas de duração, com agendamento de datas para entrega e retirada dos questionários enviados às enfermeiras.

Constituiu-se, então, um instrumento com 16 áreas e 3 níveis de cuidado por complexidade assistencial. Cada um dos níveis possui gradação de 1 a 3 apontando a intensidade crescente de complexidade, de forma que o valor 1 corresponde ao menor nível de atenção de enfermagem e o valor 3 ao nível máximo de complexidade assistencial: termorregulação, peso, atividade espontânea, reação à estímulos, cor da pele, tonicidade muscular, nutrição e hidratação, eliminações, oxigenação, integridade cutâneo-mucosa, cuidado corporal, controle de sinais vitais (T, FR, FC, PA, Saturação de O2), controle de drenos e sondas, controle de cateteres, terapêutica medicamentosa, educação à saúde.

O recém-nascido foi classificado em todas as áreas de cuidados em um dos 3 níveis na opção que melhor descreve o seu estado. Os valores obtidos individualmente, em cada área, são então somados, conduzindo a uma classe ou categoria de cuidados, que foram definidas observando as áreas de cuidados já determinadas pela pesquisadora: cuidados mínimos, cuidados intermediários, cuidados intensivos.

Obteve-se um escore padronizado que permitiu avaliar a instabilidade clínica do RN através do número de intervenções terapêuticas, da monitorização realizada em cada criança, bem como a instabilidade clínica de cada RN.

Neste momento, ainda não temos um escore sobre o qual possamos acreditar que, isoladamente e em termos absolutos, possa nos fornecer a chave que necessitamos para avaliar nosso desempenho. Gostaríamos de ter um instrumento que pudesse nos indicar de maneira correta e sensível a eficiência e a qualidade da nossa atuação clínica.

Proposta final do instrumento para classificação de recém-nascidos de acordo com o grau de dependência

Após as devidas alterações sugeridas pelas juízas, o instrumento para classificação de recém-nascidos de acordo com o grau de dependência, agora validado, adquiriu a seguinte forma final:

I- TERMORREGULAÇÃO (capacidade de manutenção da temperatura corporal estável, com gasto calórico e consumo de oxigênio mínimos, para uma adaptação extrauterina bem sucedida)

1- RNs que estão em berço comum (BC) ou em berço aquecido (BA)

2- RNs que estão em BC, BA ou em incubadora (I)

3- RNs que estão em BA ou em I

II - PESO (necessidade de controle de peso para comparar o peso diário com o peso ao nascer e com o peso do dia anterior, auxiliando na avaliação das condições nutricionais e riscos potenciais decorrentes do peso de nascimento)

1- Superior a $2500 \mathrm{~g}$

2- Superior a $1000 \mathrm{~g}$

3- Superior a $500 \mathrm{~g}$ ou independente do peso

III-ATIVIDADE ESPONTÂNEA (habilidade em manter o estado de consciência, a resposta comportamental aos estímulos sensoriais, proprioceptivos, bioquímicos, térmicos e mecânicos e parâmetros fisiológicos adequados para uma adaptação extrauterina bem sucedida) 
1- RNs ativos e/ou hiperativos

2- RNs ativos e/ou hipoativos e/ou hiperativos

3- RNs ativos e/ou hipoativos e/ou hiperativos e/ou RNs submetidos à sedação

IV - REAÇÃO À ESTÍMULOS (capacidade de responder a estímulos sensoriais, proprioceptivos, bioquímicos, térmicos e mecânicos adequados para uma adaptação extrauterina bem sucedida)

1 - RNs reativos e/ou hiperreativos

2- RNs reativos e/ou hiporeativos e/ou hiperreativos

3- RNs reativos e/ou arreativos e/ou hiporeativos e/ ou hiperreativos

V - COR DA PELE (capacidade de manter pele e mucosas coradas adequadas para uma adaptação extrauterina bem sucedida)

1 - RNs corados, variando de acordo com os antecedentes raciais, acianóticos, quando mantidos em ambientes termonêutros e/ou pletóricos e/ou com icterícia fisiológica

2- RNs com coloração cianótica e/ou pletora e/ou palidez e/ou descorados e/ou com icterícia patológica zonas I a III

3- RNs com coloração cianótica e/ou matizados e/ ou palidez e/ou pletora e/ou descorados e/ou com icterícia patológica zonas IV e $\mathrm{V}$

VI - TONICIDADE (capacidade de manter-se com tônus muscular vigoroso adequado a uma adaptação extrauterina bem sucedida)

1- Tônus muscular pronunciado, vigoroso, com movimentos espontâneos freqüentes e regulares

2- Tônus muscular reduzido e/ou com movimentos involuntários de tremores ou abalos e/ou com movimentos espontâneos leves e diminuídos

3- Tônus muscular flácido e/ou espástico com pouco ou nenhum movimento espontâneo e/ou com movimentos trêmulos, irregulares e assimétricos

VIII - ELIMINAÇÕES (habilidade em manter eliminações urinária e intestinal espontânea com auxílio de terceiros ou por drenos e estoma)

1- Eliminações presentes e com características dentro da normalidade

2- Eliminações presentes, com alterações dos padrões da normalidade (oligúria e/ou poliúria, aumento ou diminuição da frequiência das fezes e alteração da consistência e/ou coloração), com ostomias, controle das eliminações por peso de fralda, saco coletor

3- Apresenta alterações nos padrões das eliminações, com ostomias, controle das eliminações por peso de fralda e/ou presença de cateter vesical e/ou saco coletor
IX - OXIGENAÇÃO (aptidão em manter a permeabi-lidade das vias aéreas e o equilíbrio nas trocas gasosas por si mesmo ou com auxílio da equipe de enfermagem e/ou de equipamentos)

1 - RNs não dependentes de oxigênio (O2)

2- RNs submetidos a oxigenoterapia na incubadora ou por cateter nasal ou halo ou nebulização contínua

3- RNs submetidos a halo ou nebulização contínua ou CPAP nasal ou ventilação pulmonar mecânica

X-INTEGRIDADE CUTÂNEO-MUCOSA (capacidade de manter pele e mucosas sem danificação ou destruição)

1 - Integridade preservadas da pele e mucosas.

2- Presença de incisões cirúrgicas e mucosas com alteração na integridade

3- Presença de incisões cirúrgicas e soluções de continuidade da pele e mucosas

XI - CUIDADO CORPORAL (capacidade de manter a higiene pessoal, vestuário)

1- Banho de imersão, higiene perineal, oral e ocular e trocas de fralda e vestimenta realizadas pela mãe/família sob orientação e supervisão direta da equipe de enfermagem

2- Banho no leito ou de imersão, higiene perineal, oral e ocular realizados pela equipe de enfermagem com acompanhamento da mãe e trocas de fralda e vestimenta realizadas pela mãe/família com supervisão direta da equipe de enfermagem

3- Banho no leito, higiene perineal, oral e ocular e trocas de fralda e vestimenta realizadas pela equipe de enfermagem

XII - CONTROLE DE SINAIS VITAIS (SSVV) (necessidade de observação e controles dos parâmetros vitais temperatura $(\mathrm{T})$, freqüência respiratória $(\mathrm{FR})$, frequiência cardíaca (FC), pressão arterial (PA), saturação de $\mathrm{O} 2$

1- Controle $\mathrm{T}$ de 6 em 6 horas

2- Controle de T, FR, FC, PA, saturação de O2 6 em 6 horas ou de 4 em 4 horas

3- Controle de T, FR, FC, PA, saturação de $\mathrm{O} 2$ inferior a 4 horas

XIII - CONTROLE DE SONDAS E DRENOS (necessidade de observação e controle dos equipamentos contendo fluidos de infusão e/ou drenagem) fluidos

1 - RNs que não dispõem de equipamentos contendo

2- RNs que são submetidos à SOG ou SNG ou SNJ e/ou SVD (sonda vesical de demora) e/ou DVP (derivação ventricular peritoneal) ou DVE (derivação ventricular externa) e/ou bolsa de ostomia 
3- RNs que necessitam de SOG ou SNG ou SNJ e/ ou SVD e/ou DVP (derivação ventricular peritoneal) ou DVE (derivação ventricular externa) e/ou bolsa de ostomia e/ou dreno de tórax e/ou cânula endotraquel (CET).

\section{XIV - CONTROLE DE CATETERES VENOSOS (necessi-} dade de observação e controle dos cateteres de infusão e/ou coletas, monitorização hemodinâmica e nutrição parenteral hipertônica)

1- RNs que não utilizam

2- RNs submetidos a cateteres periféricos intermitentes de curta duração e/ou cateterização de vasos umbilicais

3- RNs que necessitam de cateterização de vasos umbilicais e/ou cateteres percutâneos ou venodissecção

XV-TERAPÊUTICA MEDICAMENTOSA (utilização dos diversos medicamentos terapêuticos - drogas, soluções, sangue e hemoderivados)

1- Medicação VO (via oral) e/ou via nasal e/ou via ocular e/ou via otológica e/ou via tópica e/ou via retal e/ ou IM (intramuscular) e/ou SC (subcutânea)

2- Medicação VO e/ou SOG ou SNG e/ou via nasal e/ou via ocular e/ou via otológica e/ou via tópica e/ou via inalatória e/ou via retal e/ou IM e/ou SC e/ou EV (endovenosa) intermitente e/ou EV contínua

3- Medicação VO e/ou SOG ou SNG e/ou via nasal e/ou via ocular e/ou via otológica e/ou via tópica e/ou via inalatória e/ou via retal e/ou IM e/ou SC e/ou EV contínua e/ou em uso de drogas vasoativas e/ou exsangüínotransfusão

XVI - EDUCAÇÃO À SAÚDE (habilidade, confiança e segurança da mãe/família para dispensar cuidados adequados para a manutenção dos hábitos de saúde pessoais e/ou ambientais dos RNs)

1- Orientações da equipe de enfermagem à mãe/família para prestar o cuidado com aceitação ou não das informações recebidas, ainda que estejam com dificuldade de compreensão, apreensivos ou resistentes

2- Orientações da equipe de enfermagem à mãe/família para prestar o cuidado com aceitação ou não das informações recebidas, mas com elevada apreensão e resistência e com fortes reações emocionais sobre o cuidado de higiene, alimentação, afetividade, cuidados peri e pós-operatórios, os procedimentos diagnósticos, as rotinas hospitalares e as atividades diárias

4- Orientações da equipe de enfermagem à mãe/família com compreensão ou não das informações recebidas, sem aceitá-las e com severas reações emocionais sobre o cuidado de higiene, alimentação, afetividade, cuidados peri e pós-operatórios, os procedimentos diagnósticos, as rotinas hospitalares e as atividades diárias.

\section{CONSIDERAÇÕES FINAIS}

A reconstrução do instrumento para classificação de recém-nascidos de acordo com o grau de dependência foi baseada nas necessidades individualizadas de cuidado de enfermagem ${ }^{(3)}$.

A elaboração de um instrumento de classificação específico ao recém-nascido fará com que a prática administrativa destas unidades contemple a alocação de recursos humanos e melhor monitorização da produtividade e dos custos da assistência de enfermagem ${ }^{(4)}$. $\mathrm{O}$ instrumento atua como um norteador das necessidades de cuidado, evitando a tendência de se enquadrar o neonato dentro de uma categoria de cuidado não compatível com suas necessidades, sub ou superestimando o seu nível de complexidade assistencial.

A validade do conteúdo do instrumento foi analisada por cinco juízas. O julgamento feito por estas profissionais de enfermagem demonstrou concordância quanto à manutenção da 16 áreas de cuidado propostas na composição do instrumento. Foi evidenciada a necessidade de algumas reformulações no conteúdo, no sentido de se destacar alguns procedimentos de enfermagem e uso de equipamentos que não haviam sido contemplados, bem como a inclusão e exclusão de outros considerados pertinentes.

Em sua grande maioria, as juízas consideraram existir clareza e nível de complexidade assistencial crescente nos indicadores críticos apresentados, sugerindo algumas modificações quanto a colocação das gradações bem como inclusão e exclusão de outras gradações quanto à suficiência do cuidado.

A aplicação da técnica Delphi ${ }^{(15)}$ permitiu que profissionais de enfermagem com experiências diversificadas pudessem contribuir para a reconstrução do instrumento apontando aspectos que consideram de relevância para a assistência de enfermagem. A participação deste grupo de enfermeiras, com amplo conhecimento e vivência na área de enfermagem neonatal, tornou o instrumento validado, quanto ao conteúdo, às reais necessidades da enfermeira que atua no processo de cuidar do recémnascido.

O sistema de classificação é, também, uma importante contribuição para o ensino de graduação, uma vez que favorece o desenvolvimento das atividades do processo de ensino-aprendizagem, num contexto da gradação das experiências vivenciadas pelo estudante ${ }^{(8)}$.

Esta pesquisa ateve-se à reconstrução de um instrumento de classificação de recém-nascidos e sua validação efetuou-se apenas quanto ao aspecto do conteúdo. Propomo-nos a dar continuidade, em pesquisas futuras, no sentido de processar a validação clínica desse instrumento para a verificação de sua aplicabilidade na prática assistencial. 


\section{REFERÊNCIAS}

1. Scochi CGS. A organização do trabalho e a prática de enfermagem em unidades neonatais de diferentes níveis de complexidade. Regional de Ribeirão Preto ERSA - 50 [tese]. Ribeirão Preto (SP): Escola de Enfermagem de Ribeirão Preto, Universidade de São Paulo; 1993.

2. Toledo RB, Fugulin FMT. Sistema de classificação de pacientes: construção de um instrumento para uma unidade de neonatologia. [Apresentado ao Programa de Aprimoramento de Enfermagem em Neonatologia do Hospital das Clínicas da Faculdade de Medicina da USP; 2000; São Paulo].

3. Bochembuzio L. Instrumento para classificação de recém-nascidos de acordo com o grau de dependência [tese]. São Paulo (SP): Escola de Enfermagem da Universidade de São Paulo; 2002.

4. Faro ACM. Técnica Delphi na validação das intervenções de enfermagem. Rev Esc de Enferm USP. 1997;31(2):259-73.

5. Novaes HM. Padrões e indicadores de qualidade para hospitais. Brasil. Washington: OPAS; 1994.

6. Conselho Federal de Enfermagem. Resolução 189/96. Estabelece parâmetros para dimensionamento do quadro de profissionais de enfermagem nas instituições de saúde. In: Conselho Regional de Enfermagem de São Paulo. Documentos básicos de enfermagem: enfermeiros, técnicos e auxiliares. São Paulo; 1997. p. $177-80$.

7. Connor RJ Effective use of nursing resources: a research report. Hospitals. 1961;35 (5):30-9.

8. Fugulin FMT. Implantação do sistema de classificação de pacientes na unidade de clínica médica do Hospital Universitário de São Paulo. Rev Med HU-USP. 1994;4 (1-2):63-8.

9. American Academy of Pediatrics. Standards and recomendations for hospital care of newborn infants. 6th ed. Illinois; 1977.

10. Viegas D. A organização da assistência perinatal. In: Viegas D, Vilhena-Moraes R, editores. Neonatologia clínica e cirúrgica. São Paulo: Atheneu; 1986. p. 24-7.

11. Brasil. Ministério da Saúde. Coordenação Materno-Infantil. Manual de assistência ao recém-nascido. Brasília (DF); 1994.

12. Tronchin DMR, Toma E. Estrutura e organização da unidade neonatal: aspectos de enfermagem. In: Leone CR, Tronchin DMR, editores. Assistência integrada ao recém-nascido. São Paulo: Atheneu; 1996. p. 3-20.

13. Segre CAM, Armellini PA. Organização da assistência neonatal. In: Segre CAM, Armellini PA, Marino WT, editores. Recémnascido. São Paulo: Sarvier; 1995. p. 585-94.
14. Cianciarullo TI.O desenvolvimento do conhecimento na enfermagem: padrões de conhecimento e sua importância para o cuidar. In: Cianciarullo TI, Gualda DMR, Melleiro MM, Anasuki MH, organizadoras. Sistema de assistência de enfermagem: evolução e tendências. São Paulo: Ícone; 2001. p. 15-28.

15. Spínola AWP. Delphos: proposta tecnológica alternativa. São Paulo: [s.n.]; 1984.

16. Perroca MG, Gaidzinski RR. Avaliação da capacidade preditiva e formato final de um instrumento para classificação de pacientes. Acta Paul Enferm. 2003;16(2):56-66.

17. Barradas GM, Carvalho WB. Métodos de avaliação prognóstica. In: Félix VN, Carvalho WB, Auler Junior JOC, Proença Filho, editores. Terapia intensiva adulto: pediatria/RN. São Paulo: Sarvier; 1997. p. 29-34.

18. Horta WA. Processo de enfermagem. São Paulo: EPU/EDUSP; 1979. 